\title{
Properties of directional couplers using photonic crystal waveguides
}

Thorhauge, Morten; Borel, Peter Ingo; Frandsen, Lars Hagedorn; Kristensen, Martin; Lavrinenko, Andrei; Chong, $\mathrm{H}$.

Published in:

Optical Fiber Communications Conference, 2003. OFC 2003

Link to article, DOI:

10.1109/OFC.2003.1248363

Publication date:

2003

Document Version

Publisher's PDF, also known as Version of record

Link back to DTU Orbit

Citation (APA):

Thorhauge, M., Borel, P. I., Frandsen, L. H., Kristensen, M., Lavrinenko, A., \& Chong, H. (2003). Properties of directional couplers using photonic crystal waveguides. In Optical Fiber Communications Conference, 2003. OFC 2003 (pp. 494-495). IEEE. https://doi.org/10.1109/OFC.2003.1248363

\section{General rights}

Copyright and moral rights for the publications made accessible in the public portal are retained by the authors and/or other copyright owners and it is a condition of accessing publications that users recognise and abide by the legal requirements associated with these rights.

- Users may download and print one copy of any publication from the public portal for the purpose of private study or research.

- You may not further distribute the material or use it for any profit-making activity or commercial gain

- You may freely distribute the URL identifying the publication in the public portal 
first describe the properties of the high index inclusions (layers or cylinders). Each high-index layer can be considered as a step index waveguide that supports bound normal modes and associated cutoff conditions for those modes. In what follows we show that the spectral minima for the light propagating in the core are in fact determined by the modal cutoff condition for the modes of these high-index layers in the cladding of the waveguide. In the case of step-index planar and cylindrical waveguides, the cutoff condition for these modes can be found analytically. At the cutoff (resonant) wavelength for a particular mode, the high-index layer becomes "transparent" and light escapes from the core resulting in minima in the transmission spectrum.

For planar and ring structures, the location of the transmission minima using this method are the same as those derived in Ref. [5] for the ARROW waveguide using a Fabry-Perot approach. This should be expected as the resonance condition for the cascaded Fabry-Perot sections matches the mode cutoff condition in the slab waveguide. The present approach, however has an advantage over the Fabry-Perot analogy when considering complex MOF structures, where the Fabry-Perot resonance conditions are no longer obvious, while the mode cutoffs are still obtainable. Because of their similarities, the planar and ring structures will be considered together.

I. Planar and ring structures (Fig. 1(a), (b))

For these geometries the cutoff conditions (and the spectral minima) for the one-dimensional structure can be found from the following equation:

$$
\tan \left(\frac{\pi d}{\lambda} \sqrt{n_{2}^{2}-n_{1}^{2}}-\frac{m \pi}{2}\right)=0
$$

and are given by

$$
\lambda_{m}=\frac{2 d}{m} \sqrt{n_{2}^{2}-n_{1}^{2}}
$$

A comparison of the predictions obtained from Eq. (2) with numerical simulations using beam propagation method, are shown in figure 2 along with a schematic of modal cutoff.

Along with verifying the accuracy of our predictions for the spectral minima, figure 2 (c) indicates that it is generally higher order modes that are excited in the high index inclusions. Although any mode is allowed by equation 2, the light is launched into the low index region, thus Snell's law determines the angles of the light in the high index areas. Because the index difference between these regions is large, the low angles necessary to excite the lowest modes cannot be reached. Thus the primary mode excited will be the lowest one possible. Higher modes can exist, but in typical launch conditions, these modes will have little energy.

11. Two-dimensional structure - cylinders

Again we look for the location of the transmission dips in the MOF to be at the mode cutoff locations. The natural modes of the most general circular cylinder with arbitrary isotropic internal and external media are given by Stratton [6]. The structure shown in Fig. 1(c) supports three mode types: TE, TM, and hybrid EH modes.

Similar to section I, we find the resonant condition as follows

$$
\lambda_{m}=\frac{2 d \sqrt{n_{2}^{2}-n_{1}^{2}}}{m+1 / 2}
$$

Figure 3 (a) shows real and imaginary parts of the effective index as a function of wavelength for the structure shown in figure $3(\mathrm{~b})$. The imaginary part corresponds to loss in the structure, which mean large $\operatorname{Im}\left(\mathrm{n}_{\mathrm{eff}}\right)$ corresponds to high loss or transmission spectrum minima. The locations predicted by Eq. (3) are compared to those obtained using the multipole method and are shown by straight lines in figure 3 (a). Also described in the figure is the resulting modal structure (longitudinal part of the Poynting vector $S_{z}$ and cross section of $S_{z}$ along $X$ axis) in the inclusions.

\section{Conclusion}

In summary, we proposed a simple analytical model to describe the spectral properties of photonic crystal waveguides and fibers with low-index core and high-index inclusions. This model suggests that the positions of spectral minima can be found by calculating the cutoff wavelength for the modes of high-index regions and therefore depend only on the mode structure of those inclusions. These predictions were compared with numerical simulations.

4. References

[1] J. C. Knight, J. Broeng, T. A. Birks, and P. St. J. Russell, "Photonic band gap guidance in optical fibers," Science 282, 1476 (1998)

[2] B. J. Eggleton, C. Kerbage, P. Westbrook, R. S. Windeler, and A. Hale, "Microstructured optical fiber devices," Optics Express 9, 698 (2001). [3] T. F. Krauss, R. M. De La Rue, "Photonic crystals in the optical regime: past, present and future," Progress in Quantum Electron. 23, 51 (1999)

[4] R. Bise, R. S. Windeler, K. S. Kranz, C. Kerbage, B. J. Eggleton, and D. J. Trevor, OFC 2002, Paper ThK3.

[5] N. M. Litchinitser, A. K. Abeeluck, C. Headley, B. J. Eggleton, "Antiresonant reflecting photonic crystal optical waveguides," Opt. Lett. 27, $1592(2002)$.

[6] J. A. Stratton, Electromagnetic theory, (McCRAW-HILL, 1941)

\section{ThI5}

$9: 45 \mathrm{AM}$

Properties of Directional Couplers using Photonic Crystal Waveguides

M. Thorhauge, P. Borel, L. Frandsen, M. Kristensen, COM, Tech. University of Denmark, Kgs. Lyngby, Denmark; A. Lavrinenko,

Belarussian State University, Minsk, Belarus; $\mathrm{H}$. Chong, University. of Glasgow, Glasgow, United Kingdom,Email:mih@com.dtu.dk.

Coupled photonic crystal waveguides have been designed and modelled with a 3D Finite-Difference-Time-Domain method, and fabricated in Silicon-on-Insulator material. Good agreement between modelled and measured results has been found.
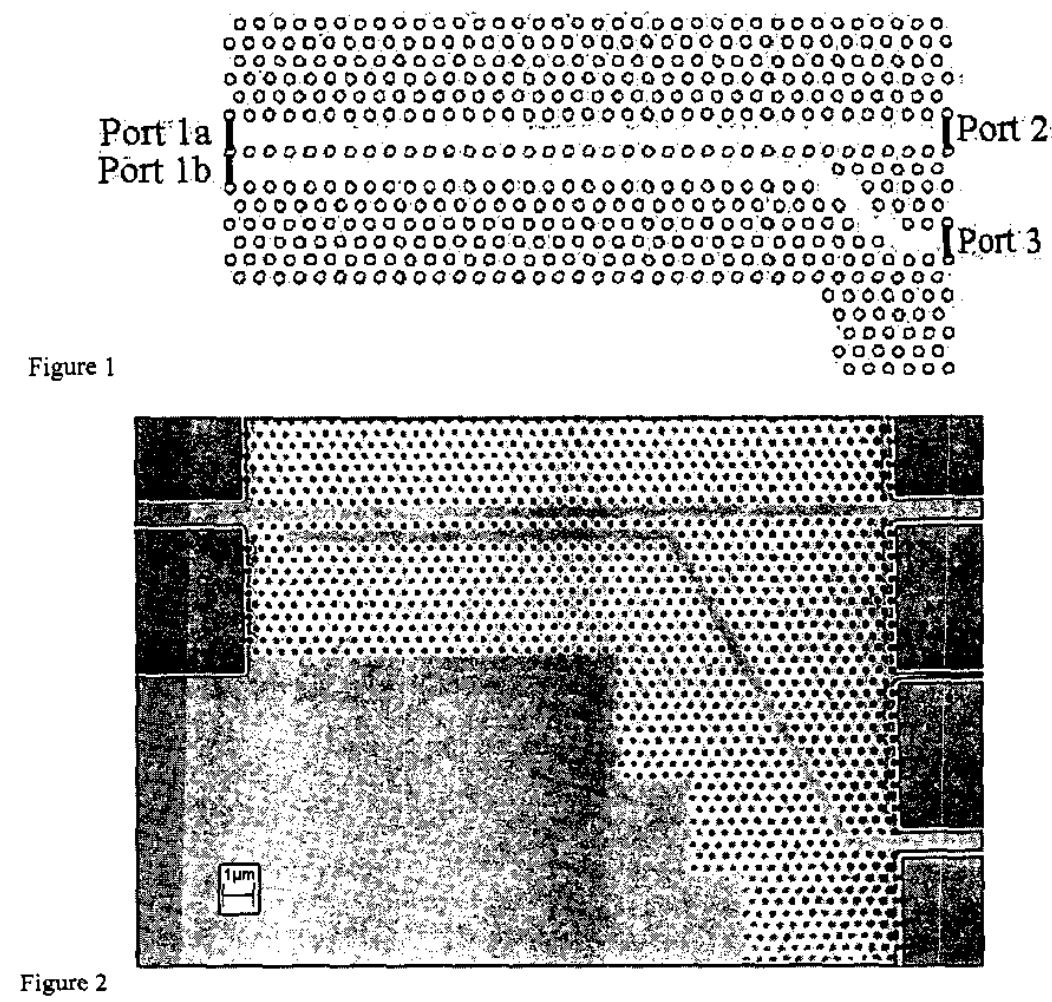

Figure 2

\section{Introduction}

Photonic Band Gap (PBG) materials [1] are important building blocks for future optoelectronic devices. In principle they can be used instead of usual index-guiding components, utilising the original properties of PBG effects in Photonic Crystals (PCs). The more confined guidance provided in PCs may be beneficial to linear and non-linear components, and the PC structure may allow novel types of components.

One of the interesting components, which can be made in $\mathrm{PC}$, is the wavelength selective waveguide coupler. Such a coupler may show new features not realisable in traditional indexguided ridge waveguide couplers due to the much higher confinement of light and the fundamentally different guiding properties.

We have done extensive modelling of such coupled waveguide structures in a 3D Finite Difference Time Domain (FDTD) scheme, and fabricated the designed components in Silicon-onInsulator (Sol). The modelled and experimental results are in good agreement.

Modelling

The software used for modelling is the Onyx-2 FDTD code [2] modified for 3D computation.

As pointed out recently by several authors [3-7] the transmission spectrum in the PBG zone is rather broad, indicating leaky mode contributions to the transmission. Propagation losses are determined by the coupling of leaky modes lying above the light cone with air and substrate radiative modes, and can therefore only be described correctly in 3D calculations.

The PC structures modelled consist of a triangular pattern of air holes made in Sol. The holes were chosen to have a normalised radius of 0.375 (radius/lattice pitch), as this gives the largest band-gap $[8]$. The holes in the calculations were given a dielectric constant $\varepsilon=1.4111$, in order to mimic the fabricated samples, which have a layer of Silica on the hole wall.

The waveguides were defined by removing one row of nearest-neighbour holes, thereby making a line defect. The layer structure for the 3D calculations consisted of $1 \Lambda$ Silica base cladding, $5 / 8 \Lambda$ Silicon core, $1 / 4 \Lambda$ Silica top cladding, and $3 / 4 \Lambda$ air, $\Lambda$ being the lattice pitch, the centre-to-centre distance between nearest-neighbour holes. 
The modelled structure had a coupling region consisting of two waveguides next to each othe separated by a single row of holes. The coupling length was $27 \Lambda$. After the coupling region one waveguide was continued for an additional $7 \Lambda$ while the other channel was led away from the first via two 60-degree bends and a short intermediate waveguide section, creating an output por separation of 5 rows, see figure 1

The bends were optimised in order to increase transmission (one hole moved) [9].

The component has four ports, two input ports la and $1 \mathrm{~b}$, and two output ports 2 and 3 . Only one input port was excited at a time, the input port no in use being blocked by three holes following the triangular patterm.

Experiment

For the experimental part two samples were fabricated with structures resembling the ones modelled (figure 1), one with port la blocked, and the othe with port $1 \mathrm{~b}$ blocked (figure 2 ).

To be able to define in- and out-coupling ridge waveguides it was necessary to separate the output ports further with a longer intermediate section in the waveguide with two sixty degree bends, so the output ports were spaced by 28 rows.

E-beam lithography was used to define the hole pattem in resist on Sol wafers. The e-beam resist acted as a mask in the subsequent transfer of the pattern to the Silicon layer by Reactive Ion Etching (RIE). After removal of the resist, the pattem was further transferred to the Silica base cladding by RIE, using the Silicon core layer as a mask. Finally, a Silica top cladding was grown on the surface of the Silicon layer by thermal oxidation. The Silica top cladding serves to make the structure vertically symmetric and more robust. The lattice pitch $\Lambda$ was chosen to $428 \mathrm{~nm}$.

Ridge waveguides gradually tapered down from 4 um to $1 \mu \mathrm{m}$ at the PC structure were used to route the light to and from the ports.

We performed transmission measurements using tapered lensed fibres to couple light into and out of the sample. The light sources consisted of unpolarised light emitting diodes with centre wavelengths at $1310 \mathrm{~nm}$ and $1550 \mathrm{~nm}$. The spectra were recorded using an ANDO AQ-6315A optical spectrum analyser. Before each measurement the coupling to the sample was optimised for maximum transmission. All measurements were normalised to a fibre-to-tapered-ridge-to-fibre measurement.

\section{Results and comparison}

For the coupler with input in port la, (see figure 1) we get the results plotted in figure 3 . This is the straight-through result with transmission measured at port 2. As the 3D FDTD model uses a TE polarised source, and the sources in the experiment were unpolarised, the measured results have been added $3 \mathrm{~dB}$ to account for this, as the TM modes are expected to be highly lossy due to the lack of band gap at the examined wavelength band $[1,8]$.

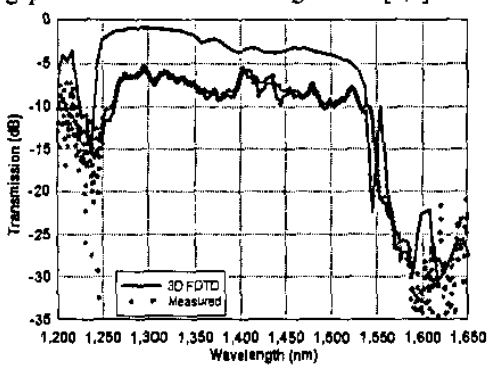

Figure 3

There is a significant resemblance between the measured and the calculated data. The trend is the same for the two sets of results. There is some difference in absolute value. The coupling loss from ridge waveguide to $P B G$ waveguide and from $P B G$ waveguide to ridge waveguide is one source of loss in the experiment, which is not present in the modelled results, and is not eliminated by normalisation to a ridge waveguide.

Some of the finer features from the measurement are missing in the computed result. This is likely caused by the difference in the output section for port 3 , where the arm is longer in the experimental sample, creating more and finer Fabry-Perot type resonances.

Due to a limited bandwidth of the source, the experimental data gets quite noisy at the sides of the spectrum.

The measured results look slightly shifted along the frequency axis when compared with the computed results. This is likely due to minor differences in the core and top cladding layer thicknesses and the hole radii between the modelled sample and the measured sample caused by insufficient precision in the fabrication. The fabrication methods are constantly being improved to reduce such differences.

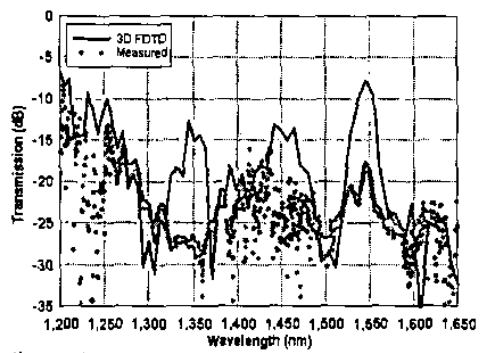

Figure 4

In figure 4 modelled and measured results for a coupler with input into port $1 \mathrm{~b}$ and transmission measured at port 2 is shown. This is the coupled result. Again a good resemblance is seen, though the calculated peak around $1350 \mathrm{~nm}$ is completely missing in the experimental data. The reason for this is currently not fully understood. Between $1400 \mathrm{~nm}$ and $1480 \mathrm{~nm}$ the measured data are quite noisy, which is due to the weakness of the source in this region together with the low level of coupling. $3 \mathrm{~dB}$ are added to the measured data here too.

Looking at figures 3 and 4 a strong dependence of coupling on frequency can be seen. Around 1560 $\mathrm{nm}$ the uncoupled transmission drops rapidly more than $20 \mathrm{~dB}$, and the structure acts as a low-pass filter. For the coupled transmission in figure 4 , the spectrum peaks around $1545 \mathrm{~nm}$ with a peak level around $10 \mathrm{~dB}$ higher than the dip at $1500 \mathrm{~nm}$. Conclusion

We have investigated coupled waveguides in photonic crystais with triangular symmetry by 3D FDTD modelling and in real life samples. We have shown, that we can fabricate samples showing qualitatively correct transmission spectra when compared to 3D FDTD modelling results. We have also shown, we can design and fabricate structures exhibiting highly wavelength selective transmission spectrum behaviour. These promising results have provided us with valuable insight into the behavior of coupled PBG waveguides, which can be used in the design and modelling of new, efficient components utilising such structures.

References

1 J. D. Joannopoulos et al, Photonic Crystals: Molding the Flow of Light, Princeton University Press (1995).

2 A. J. Ward, and J. B. Pendry, Comp. Phys. Com. $128(2000), 590$

3 N. Kawai et al, Phys. Rev. Lett., 86 (2001), 2289. 4 Y. Sugimoto et al, Appl. Phys. Lett., 79 (2001), 4286

5 Y. Sugimoto et al, Optics Lett., 27 (2002), 388. 6 T. Ochiai, and K. Sakoda, Phys. Rev. B, 63 (2001), 125107.

7 M. Loncar et al, Appl. Phys. Lett., 80 (2002), 1689

8 T. Sondergaard et al, Optics Com., 194 (2001), 341. 9 A. Talneau et al, Appl. Phys. Lett., 80 (2002) 547.
ThI6

10:00 AM

Fabrication and Characterization of Low Loss Heterostructured Photonic Crystal Waveguides

Y. Ohtera, K. Miura, H. Ohkubo, T. Sato, S. Kawakami, NICHe, Tohoku University, Sendai, Japan; T. Sato, S. Kawakami, Photonic Lattice Inc., Sendai, Japan, Email: ohtera@niche.tohoku.ac.jp.

We report a fiber butt-jointable $\mathrm{Ta}_{2} \mathrm{O}_{5} / \mathrm{SiO}_{2}$ photonic crystal waveguide with a record low-loss $(0.56 \mathrm{~dB} / \mathrm{mm})$. The waveguide has a heterostructure and is fabricated by the autocloning technol. ogy. Details are described.

1. Introduction

In recent years, a growing number of research have been carried out to establish the waveguide technologies of photonic crystals, especially the types called "line-defect waveguides," which confine light by the effect of photonic bandgap. The merits of employing such photonic crystal waveguides are the availability of strong Bragg reflections of the guided modes and sharp bends. It is expected that one needs only a few square millimeters of circuit to integrate multiple functions such as wavelength filters and multiplexers demultiplexsers. However, one of nontrivial difficulties of such waveguides is the problem how to couple to/from optical fibers: Typically, their mode spotsize is less than one micrometer, and butt-coupling results in a large loss. There is no other choice than to use a number of bulky lenses. We have so far proposed and developed a nove class of photonic crystal waveguides, which shows low-loss light propagation and fiber/PLC compatible modal fields. In this study, we redesigned the core structure and improved the waveguide performance of loss characteristics high-density circuit integration capability, and easy butt-coupling.

The following is the key of this work

(1) The minimum net propagation loss is 0.56 $\mathrm{dB} / \mathrm{mm}$ at $\lambda=1.55 \mu \mathrm{m}$

(2) The waveguide consists of a heterostructured photonic crystal. The core and the claddings over and under it have flat multilayer structure. On the other hand, the side claddings have two-dimensional periodic structure.

(3) The size of the core is $2 \mu \mathrm{m} \times 2.6 \mu \mathrm{m}$. The inplane index difference between the core and the side claddings is $3.6 \%$

(4) The spot at the waveguide end is circular and its diameter is $4.5 \mu \mathrm{m}$.

We thus report a significant advance of the waveguide as compared to our previous work [l] The new waveguide is of course compatible with devices such as resonators [1] etc.

2. Structure of the waveguide and the principle of the operation

Fig. 1 shows a schematic view of the waveguide It consists of a heterostructured photonic crystal with multiple regions having different lattice types/constants [2,3]. It is fabricated by the autocloning method [4]. As shown in Fig. 2, the waveguide has nine different regions in its cross section. In the horizontal direction, a flat region of the width of $2 \mu \mathrm{m}$ acts as a core, while wavy regions with the in-plane pitch of $1.1 \mu \mathrm{m}$ around it act as cladding. For the vertical direction, the core layers and the cladding layers have the lattice constants of $0.73 \mu \mathrm{m}$ and $0.60 \mu \mathrm{m}$, respectively, and the difference causes confinement of light. The light is confined in the center region of Fig. 2 and propagates along $\mathrm{z}$ direction. The constituen materials of the multilayer are $\mathrm{Ta}_{2} \mathrm{O}_{5}(n=2.1)$ and $\mathrm{SiO}_{2}(\mathrm{n}=1.5)$. Each region has an effective refractive index as shown in Fig. 3 for the horizontally polarized light of $\lambda=1.55 \mu \mathrm{m}$. We make use of the effect that the flat multilayer has a larger effective index than that of wavy multilayers for horizontally polarized light. In this design, the relative index difference $(\Delta)$ between the core and the vertical and horizontal claddings are approximately $0.9 \%$ and $3.6 \%$, respectively. The light is strongly confined in the in-plane direction. By making use of such large index difference, one can 